\title{
A Stay-in-a-Set Game without a Stationary Equilibrium
}

\author{
Kristoffer Arnsfelt Hansen \\ Aarhus University \\ Department of Computer Science \\ arnsfelt@cs.au.dk
}

\author{
Mikhail Raskin * \\ Technical University of Munich \\ Department of Informatics \\ raskin@mccme.ru
}

\begin{abstract}
We give an example of a finite-state two-player turn-based stochastic game with safety objectives for both players which has no stationary Nash equilibrium. This answers an open question of Secchi and Sudderth.
\end{abstract}

\section{Introduction}

Stochastic games provide a general model for studying dynamic interactions between players whose actions affect the state of the environment. The change in state is described by a probability distribution called the law of motion. The first such games were introduced by Shapley [17]. We may view his model as discrete-time finite two-player zero-sum games, where players receive immediate payoff in each round of play and discount future payoffs. Shapley proved that in such games optimal stationary (or, memoryless) strategies exists. The initial model of Shapley has since been extensively extended and studied in many variations. With each model the main question is existence of optimal strategies or a Nash equilibrium. Next, it is of interest how complicated such strategies must be. We shall limit our discussion to discrete-time games having an arbitrary but finite number of states.

Everett [6] defined recursive games, where players only receive a (possibly) non-zero payoff when play terminates by entering special absorbing states. These payoffs are also called terminal payoffs. While players are no longer guaranteed to have optimal strategies, Everett proved that they do have $\varepsilon$ optimal stationary strategies. Gillette [9] considered finite two-player zero-sum games where players again receive immediate payoffs each round of play, but now evaluate their payoff as the average of the immediate payoffs received (limit average payoff). Here players are no longer guaranteed to have $\varepsilon$-optimal stationary strategies, but as shown by Mertens and Neyman [15] they do have $\varepsilon$-optimal strategies. An even more general result was obtained by Martin [13] showing that for two-player zero-sum games where payoffs are Borel measurable functions of the history of play, the players have $\varepsilon$-optimal strategies. Here the extension from deterministic games (i.e. games having a deterministic law of motion) to the general case is due to an observation of Maitra and Sudderth [11].

For non-zero sum games much less is known. For discounted payoffs, a Nash equilibrium exists in stationary strategies as shown by Fink [7] and Takahashi [19]. The existence of $\varepsilon$-Nash equilibrium in recursive games is an open problem, even for three players. In addition, Flesch, Thuijsman and Vrieze [8] gave an example of a two-player recursive game without stationary $\varepsilon$-Nash equilibrium. Vieille [21, 22] proved existence of $\varepsilon$-Nash equilibrium in every two-player game with limit-average payoff.

\footnotetext{
* The author has received funding from the European Research Council (ERC) under the European UnionâĂŹs Horizon 2020 research and innovation programme under grant agreement No 787367 (PaVeS) and from the French National Research Agency (ANR project GraphEn / ANR-15-CE40-0009).
}

J. Leroux and J.-F. Raskin (Eds.): Tenth International Symposium on Games, Automata, Logics, and Formal Verification (GandALF'19).

EPTCS 305, 2019, pp. 83-90 doi 10.4204/EPTCS.305.6

(c) K. A. Hansen \& M. Raskin 
Mertens and Neyman (cf. [14]) showed, using the celebrated determinacy result by Martin [12], that an $\varepsilon$-Nash equilibrium exists in any turn-based (i.e. perfect information) game with Borel payoff functions. Later this was observed again by Chatterjee et al. [5]. When the payoff function has finite range, an actual Nash equilibrium exists. This is particularly the case of deterministic games where the payoff function is the indicator function of a Borel set. We refer to the indicator function of a Borel set as well as the set itself as a Borel winning set or Borel objective.

The most basic of these are given by the open and closed sets. Given a set of states $T$, the reachability objective Reach $(T)$ given by $T$ consists of the histories of play that visit a state in $T$. The safety objective Safe $(T)$ given by $T$ consists of the histories of play that stays within the states in $T$. These winning sets are the open and closed Borel objectives typically studied, and they have applications in the verification and synthesis of reactive systems [4].

Games where the players have reachability or safety objectives are closely related to recursive games. First note that for a given recursive game, after normalizing all payoffs to be in the range $[-1,1]$, every terminal payoff vector can be written as a convex combination of payoff vectors having only entries from the set $\{-1,0,1\}$. This means that any absorbing state can be replaced by a set of absorbing states where all players have payoffs in the set $\{-1,0,1\}$ as well by modifying the (probabilistic) law of motion accordingly. Then, if a player only receive terminal payoffs from the set $\{-1,0\}$, this is equivalent to a safety objective, and likewise if a player only receive terminal payoffs from the set $\{0,1\}$, this is equivalent to a reachability objective.

Secchi and Sudderth [16] considered the class of games where each player has a safety objective, and called these games for stay-in-a-set games. For these games they proved existence of a Nash equilibrium in any (finite) stay-in-a-set game. The equilibrium strategies are not stationary but prescribe, as a function of the set of the players whose safety objective has not yet been violated, a stationary strategy profile. This means that for $n$ players just $n$ bits of (shared) memory are needed for implementing a Nash equilibrium. A natural open question raised by Secchi and Sudderth was then existence of a stationary Nash equilibrium. We give an example of a two-player game without a stationary Nash equilibrium. Our game is furthermore turn-based. By example we also illustrate the Nash equilibria obtained from the proof of Secchi and Sudderth. They rely crucially on the willingness of the second player to change strategy after already having lost. In our example it is not necessary to remember whether the first player has lost, so a single bit of shared memory is enough for implementing the equilibrium. Finally we note that players do have a stationary $\varepsilon$-Nash equilibrium.

It is necessary that our example game is not deterministic. In fact, in every deterministic two-player turn-based games, where each player has a reachability or a safety objective, a Nash equilibrium exists in positional (i.e. pure and memoryless) strategies. This follows from the fact that two-player zero-sum games with a reachability and safety objective are positionally determined. Thus in the non-zero sum game it is either the case that one of the two players may guarantee a win (and relative to that we let the other player play optimally) or it is the case that both players can ensure that the opponent loses.

\section{The game}

The game $\mathscr{G}$ we consider is played by two players each taking turns in choosing whether to continue the game or to attempt to quit the game, with Player 1 making the first choice. A choice of the quit action by one of the players is successful with probability $3 / 4$, and otherwise the game continues with the other player as before. If Player 1 makes the choice to quit both players win with probability $1 / 2$ and both players lose with the remaining probability 1/4. If Player 2 makes the choice to quit both players win with probability $1 / 4$ and both players lose with the remaining probability $1 / 2$. Finally, Player 2 is 
incentivized to choose quit by having the continue action of Player 2 lead to a loss for Player 2 with probability $1 / 4$. Infinite play leads to Player 1 winning (and Player 2 losing with probability 1). This leads to a discontinuity in the payoff function of Player 1, which is crucial for our example.

The game $\mathscr{G}$ is illustrated in Figure 1 and is modeled with a set of 5 states $\{1,2, W, L, L 2\}$, with Player 1 controlling state 1 , Player 2 controlling state 2 . State $L 2$ exists merely to enforce a loss to Player 2, whereas the states $W$ and $L$ are winning and losing states of both players, respectively. The game $\mathscr{G}$ is a stay-in-a-set game with the safe sets of the two players being $G_{1}=\{1,2, W, L 2\}$ and $G_{2}=$ $\{1,2, W\}$, respectively. The diamond-shaped nodes in Figure 1 are used to indicate the probabilistic transitions.

A stationary strategy profile $\sigma$ in $\mathscr{G}$ can be described by a pair of probabilities $\left(p_{1}, p_{2}\right)$, where $p_{i}$ is the probability that Player $i$ chooses the quit action $q$, when in state $i$ (and thus $\left(1-p_{i}\right)$ is the probability that Player $i$ chooses the continue action $c$, when in state $i$ ).

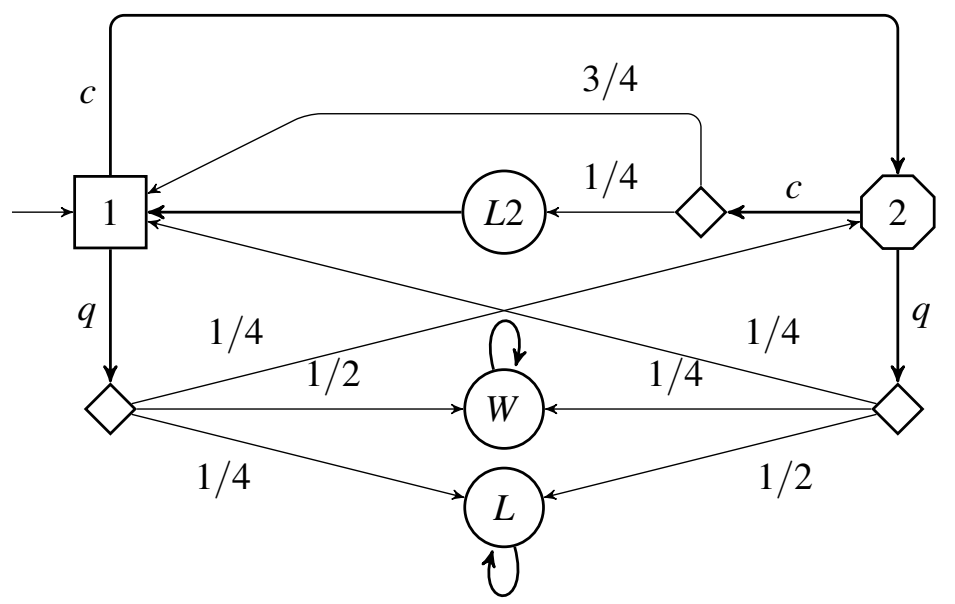

Figure 1: The game $\mathscr{G}$.

\subsection{No stationary Nash equilibrium}

We give here a simple analysis showing that no stationary Nash equilibrium exists in $\mathscr{G}$. We can place all plays of $\mathscr{G}$ in 3 groups. Group 1 are plays where Player 1 quits successfully, group 2 are plays where Player 2 quits successfully, and group 3 are plays that never reach $W$ or $L$.

Consider a stationary strategy profile $\sigma$ given by $\left(p_{1}, p_{2}\right)$. When $p_{1}=p_{2}=0$ the play belongs to group 3, where Player 1 wins and Player 2 loses with probability 1 . When $p_{1}>0$ or $p_{2}>0$ the play belongs to group 1 or group 2 with probability 1 . The players both prefer a play from group 1 where Player 1 is the player to quit successfully.

Suppose that $\sigma$ is a Nash equilibrium. If $p_{2}=0$, then the only best reply of Player 1 is to have $p_{1}=0$, since otherwise $L$ is reached with positive probability. But if also $p_{1}=0$, Player 2 loses with probability 1 , whereas $p_{2}>0$ would lead to reaching $W$ with positive probability. This rules out having $p_{2}=0$ in a Nash equilibrium.

Suppose now $p_{2}>0$, which means that the play belongs to group 1 or group 2 with probability 1 . The probability that the play belongs to group 1 strictly increases with $p_{1}$, and it follows that we must have $p_{1}=1$. But this is also not a Nash equilibrium, as Player 2 would then be better off having $p_{2}=0$. Indeed, let us consider a play from state 2 until the play either returns to state 2 , reaches state $W$ before 
returning to state 2 , or reaches state $L$ or state $L 2$ before returning to state 2 . We denote these events a return, a win, or a loss.

The quit action for Player 2 has probability $1 / 2+(1 / 4)(1 / 4)=9 / 16$ of a loss, probability $1 / 4+$ $(1 / 4)(1 / 2)=6 / 16$ of a win and $(1 / 4)(1 / 4)=1 / 16$ of a return. The continue action has probability $1 / 4+(3 / 4)(1 / 4)=7 / 16$ of a loss, probability $(3 / 4)(1 / 2)=6 / 16$ of a win and $(3 / 4)(1 / 4)=3 / 16$ of a return. Since a return is better than a loss for Player 2, this rules out $p_{2}>0$ in a Nash equilibrium as well.

\subsection{Detailed payoff analysis}

For $i, j \in\{1,2\}$, let $u_{i, j}=u_{i, j}\left(p_{1}, p_{2}\right)$ be the payoff to Player $i$ of the strategy profile $\left(p_{1}, p_{2}\right)$ when starting play in state $j$. The payoffs satisfy the following equations

$$
\begin{aligned}
& u_{1,1}=\left(\left(1-p_{1}\right)+p_{1} / 4\right) u_{1,2}+p_{1} / 2=\left(1-3 p_{1} / 4\right) u_{1,2}+p_{1} / 2 \\
& u_{1,2}=\left(\left(1-p_{2}\right)+p_{2} / 4\right) u_{1,1}+p_{2} / 4=\left(1-3 p_{2} / 4\right) u_{1,1}+p_{2} / 4 \\
& u_{2,1}=\left(\left(1-p_{1}\right)+p_{1} / 4\right) u_{2,2}+p_{1} / 2=\left(1-3 p_{1} / 4\right) u_{2,2}+p_{1} / 2 \\
& u_{2,2}=\left(3 / 4\left(1-p_{2}\right)+p_{2} / 4\right) u_{2,1}+p_{2} / 4=\left(3 / 4-p_{2} / 2\right) u_{2,1}+p_{2} / 4
\end{aligned}
$$

and from these follows further

$$
\begin{aligned}
& u_{1,1}=\left(1-3 p_{1} / 4\right)\left(1-3 p_{2} / 4\right) u_{1,1}+\left(1-3 p_{1} / 4\right) p_{2} / 4+p_{1} / 2 \\
& u_{2,1}=\left(1-3 p_{1} / 4\right)\left(3 / 4-p_{2} / 2\right) u_{2,1}+\left(1-3 p_{1} / 4\right) p_{2} / 4+p_{1} / 2
\end{aligned}
$$

When both $p_{1}=0$ and $p_{2}=0$ we have that $u_{1,1}=u_{1,2}=1$ and $u_{2,1}=u_{2,2}=0$. When at least one of $p_{1}>0$ or $p_{2}>0$ holds, we can solve for $u_{1,1}$, and likewise we can always solve for $u_{2,1}$ to obtain

$$
\begin{aligned}
u_{1,1} & =\frac{\left(1-3 p_{1} / 4\right) p_{2} / 4+p_{1} / 2}{1-\left(1-3 p_{1} / 4\right)\left(1-3 p_{2} / 4\right)}=\frac{\left(8-3 p_{2}\right) p_{1}+4 p_{2}}{\left(12-9 p_{2}\right) p_{1}+12 p_{2}} \\
& =\frac{\left(4-3 p_{1}\right) p_{2}+8 p_{1}}{\left(12-9 p_{1}\right) p_{2}+12 p_{1}} \\
u_{2,1} & =\frac{\left(1-3 p_{1} / 4\right) p_{2} / 4+p_{1} / 2}{1-\left(1-3 p_{1} / 4\right)\left(3 / 4-p_{2} / 2\right)}=\frac{\left(4-3 p_{1}\right) p_{2}+8 p_{1}}{\left(8-6 p_{1}\right) p_{2}+9 p_{1}+4} \\
& =\frac{\left(8-3 p_{2}\right) p_{1}+4 p_{2}}{\left(9-6 p_{2}\right) p_{1}+8 p_{2}+4}
\end{aligned}
$$

Using $\frac{\partial}{\partial x} \frac{a x+b}{c x+d}=\frac{a d-b c}{(c x+d)^{2}}$, we find that

$$
\operatorname{sgn}\left(\frac{\partial}{\partial p_{1}} u_{1,1}\right)=\operatorname{sgn}\left(48 p_{2}\right)=1 \text { for all } p_{2}>0
$$

And likewise $\operatorname{sgn}\left(\frac{\partial}{\partial p_{2}} u_{2,1}\right)=\operatorname{sgn}\left(\left(4-3 p_{1}\right)\left(4-7 p_{1}\right)\right)$, which means that

$$
\operatorname{sgn}\left(\frac{\partial}{\partial p_{2}} u_{2,1}\right)=\left\{\begin{aligned}
1 & \text { if } p_{1}<\frac{4}{7} \\
0 & \text { if } p_{1}=\frac{4}{7} \\
-1 & \text { if } p_{1}>\frac{4}{7}
\end{aligned}\right.
$$


The function $u_{2,1}$ is continuous in the entire domain, whereas the function $u_{1,1}$ has a single discontinuity when $p_{1}=p_{2}=0$. Note that $u_{1,1}\left(p_{1}, 0\right)=\frac{2}{3}$ for all $p_{1}>0$, and $u_{1,1}\left(0, p_{2}\right)=\frac{1}{3}$ for all $p_{2}>0$.

The best replies of the players are as follows. If $p_{2}=0$, the only best reply of Player 1 is to have $p_{1}=0$, giving $u_{1,1}=1$. If $p_{2}>0$, the only best reply of Player 1 is to have $p_{1}=1$, giving $u_{1,1}=\frac{8+p_{2}}{12+3 p_{2}}$. If $p_{1}<4 / 7$, the only best reply for Player 2 is to have $p_{2}=1$, giving $u_{2,1}=\frac{4+5 p_{1}}{12+3 p_{1}}$. When $p_{1}=4 / 7$, Player 2 has no preferred action. Finally, if $p_{1}>4 / 7$ the only best reply of Player 2 is to have $p_{2}=0$, giving $u_{2,1}=\frac{8 p_{1}}{4+9 p_{1}}$.

\subsection{Nash equilibria}

We give here two examples of Nash equilibria in the game following the general result of Secchi and Sudderth [16]. The idea is that once Player 2 has lost by entering state $L 2$ the incentive of Player 2 is removed and all strategies are equally good.

Suppose first that Player 2 commits to always playing the continue action after entering state $L 2$. The best reply of Player 1 is then to always play the continue action as well, ending up with payoff 1 . We may thus consider the modified game $\mathscr{G}^{\prime}$ that stops when entering $L 2$ upon which Player 1 receives payoff 1. This lead to the modified equation

$$
u_{1,2}=\left(3 / 4\left(1-p_{2}\right)+p_{2} / 4\right) u_{1,1}+1 / 4=\left(3 / 4-p_{2} / 2\right) u_{1,1}+1 / 4
$$

giving

which solves to

$$
u_{1,1}=\left(1-3 p_{1} / 4\right)\left(3 / 4-p_{2} / 2\right) u_{1,1}+\left(1-3 p_{1} / 4\right) / 4+p_{1} / 2
$$

$$
u_{1,1}=\frac{\left(1-3 p_{1} / 4\right) / 4+p_{1} / 2}{1-\left(1-3 p_{1} / 4\right)\left(3 / 4-p_{2} / 2\right)}=\frac{5 p_{1}+4}{\left(9-6 p_{2}\right) p_{1}+4+8 p_{2}}
$$

and we see that $\operatorname{sgn}\left(\frac{\partial}{\partial p_{1}} u_{1,1}\right)=\operatorname{sgn}\left(64 p_{2}-16\right)$.

A Nash equilibria is thus that the players play the quit action with probabilities $p_{1}=4 / 7$ and $p_{2}=1 / 4$ respectively until state $L 2$ is reached and after which both players play the quit action with probability $p_{1}=p_{2}=0$. The equilibrium payoffs are $u_{1,1}=2 / 3$ and $u_{2,1}=1 / 2$.

Suppose next that Player 2 commits to always playing the quit action after entering state $L 2$. The best reply of Player 1 is then to always play the quit action as well, ending up with payoff $3 / 5$. The modified game $\mathscr{G}^{\prime}$ now has the equation

$$
\begin{array}{r}
u_{1,2}=\left(3 / 4\left(1-p_{2}\right)+p_{2} / 4\right) u_{1,1}+3 / 5\left(1-p_{2}\right) / 4+p_{2} / 4 \\
=\left(3 / 4-p_{2} / 2\right) u_{1,1}+3 / 20+p_{2} / 10
\end{array}
$$

giving

$$
u_{1,1}=\left(1-3 p_{1} / 4\right)\left(3 / 4-p_{2} / 2\right) u_{1,1}+\left(1-3 p_{1} / 4\right)\left(3 / 20+p_{2} / 10\right)+p_{1} / 2
$$

which solves to

$$
u_{1,1}=\frac{\left(1-3 p_{1} / 4\right)\left(3 / 20+p_{2} / 10\right)+p_{1} / 2}{1-\left(1-3 p_{1} / 4\right)\left(3 / 4-p_{2} / 2\right)}=\frac{\left(31-6 p_{2}\right) p_{1}+8 p_{2}+12}{5\left(9-6 p_{2}\right) p_{1}+8+4 p_{2}}
$$

We find that $\operatorname{sgn}\left(\frac{\partial}{\partial p_{1}} u_{1,1}\right)=\operatorname{sgn}\left(1120 p_{2}+80\right)=1$, which means that the best reply of Player 1 is always to play the quit action, and in turn the best reply of Player 2 to that is to always play the continue action.

A Nash equilibria is thus that the players play the quit action with probabilities $p_{1}=1$ and $p_{2}=0$ respectively until state $L 2$ is reached and after which Player 2 changes to playing the quit action with probability $p_{2}=1$ as well. The equilibrium payoffs are here $u_{1,1}=\frac{43}{65}=\frac{2}{3}-\frac{1}{195}$ and $u_{2,1}=\frac{8}{13}=\frac{1}{2}+\frac{3}{26}$. 


\subsection{Stationary $\varepsilon$-Nash equilibrium}

Whereas we have shown that the game $\mathscr{G}$ has no stationary Nash equilibrium, it does have $\varepsilon$-Nash equilibria, for any $\varepsilon>0$.

When $\varepsilon<1 / 3$ no $\varepsilon$-Nash equilibrium can have $p_{2}=0$. Indeed, then the only $\varepsilon$-best reply of Player 1 would be the actual best reply having $p_{1}=0$. To that, any $\varepsilon$-best reply of Player 2 must have $p_{2}>0$, when $\varepsilon<1 / 3$.

A few examples of $\varepsilon$-Nash equilibria are $\sigma_{1}$ given by $p_{1}=1$ and $p_{2}=\varepsilon, \sigma_{2}$ given by $p_{1}=\frac{4}{7}-\varepsilon$ and $p_{2}=\varepsilon$, and $\sigma_{3}$ given by $p_{1}=\frac{4}{7}+\varepsilon$ and $p_{2}=\varepsilon$. We omit the simple task of verifying that these are indeed $\varepsilon$-Nash equilibria. In $\sigma_{1}$ the payoffs are $u_{1,1}=\frac{2}{3}-O(\varepsilon)$ and $u_{2,1}=\frac{8}{13}-O(\varepsilon)$, and in both $\sigma_{2}$ and $\sigma_{3}$ the payoffs satisfy $u_{1,1}=\frac{2}{3}-O(\varepsilon)$ and $u_{2,1}=\frac{1}{2}-O(\varepsilon)$. We note that Player 1 is playing the best reply in $\sigma_{1}$, but is $\left(\frac{3}{7}-\varepsilon\right)$-far from the best reply $p_{1}=1$ in $\sigma_{2}$ and $\sigma_{3}$. Player 2 is playing $\varepsilon$-close to the best reply $p_{2}=0$ in $\sigma_{1}$ and $\sigma_{3}$, but $(1-\varepsilon)$-far from the best reply $p_{2}=1$ in $\sigma_{2}$.

\section{Conclusion and Further Problems}

We have given a simple example of a two-player turn-based game with safety objectives for both players without a stationary Nash equilibrium. A remaining open question is the existence of a stationary $\varepsilon$-Nash equilibrium when players have safety objectives, even in the case of two-player turn-based games.

Several related open questions concern games with reachability objectives or with combinations of reachability and safety objectives. We first consider the setting where all players have reachability objectives, also called reach-a-set games [5]. Flesch, Thuijsman and Vrieze [8] give an example of a three-player recursive game with non-negative payoffs with no stationary $\varepsilon$-Nash equilibrium. The game is furthermore deterministic. Simon [18] gave an example of a two-player recursive game with non-negative payoffs with no stationary $\varepsilon$-Nash equilibrium. These both give examples of reach-a-set games without stationary $\varepsilon$-Nash equilibria by the general method of simulating terminal payoffs with the probabilistic law of motion. The example of Flesch, Thuijsman and Vrieze is however such that the terminal payoff vectors satisfy that either none or precisely two players receive a strictly positive payoff. The payoff vectors where two players receive strictly positive payoff can (after scaling) be constructed as unique equilibrium payoffs of win-lose bimatrix games 1 . This then results in a three-player deterministic reach-a-set game with no stationary $\varepsilon$-Nash equilibrium.

For two-player games, it was erroneously claimed (cf. [3]) first by Chatterjee et al. [5] and later again by Ummels and Wojtczak [20] that a simple adaptation of an example of a zero-sum game of Everett resulted in a deterministic reach-a-set game without a Nash equilibrium. Thus it remains an open question whether every deterministic two-player reach-a-set game has a Nash equilibrium. It is also an open problem whether every deterministic two-player reach-a-set game has a stationary $\varepsilon$-Nash equilibrium. Boros and Gurvich [1] and Kuipers et al. [10] give an example of a three-player turn-based recursive game with non-negative payoffs that has no stationary Nash equilibrium. Do every two-player turn-based reach-a-set game have a stationary Nash equilibrium?

Little is known when some players have a reachability objective and some players a safety objectives. In the two-player zero-sum case an example of Everett [6] shows that optimal strategies, and hence a Nash equilibrium, may fail to exist. On the other hand an $\varepsilon$-optimal stationary equilibrium always exists. Do every two-player game where one player has a reachability objective and one player a safety objetive

\footnotetext{
${ }^{1}$ These payoff vectors are $(1,1)$ and $(3,1)$. It is easy to construct two $4 \times 4$ bimatrix games with only payoffs from the set $\{0,1\}$ in which the unique equlibrium payofff vectors are $\left(\frac{1}{4}, \frac{1}{4}\right)$ and $\left(\frac{3}{4}, \frac{1}{4}\right)$, respectively, which may replace $(1,1)$ and $(3,1)$
} 
always have a stationary $\varepsilon$-Nash equilibrium? In the case of turn-based games, it is an open problem whether every three-player deterministic game has a stationary Nash equilibrium. An example given by Boros et al. [2] appears to be close to answer this question. Namely, Boros et al. construct a three-player deterministic recursive game without a stationary Nash equilibrium, that may be realized with payoffs such that player two has only non-negative terminal payoffs and player one and player three have only non-positive terminal payoffs.

\section{References}

[1] E. Boros \& V. Gurvich (2003): On Nash-solvability in pure stationary strategies of finite games with perfect information which may have cycles. Mathematical Social Sciences 46(2), pp. 207 - 241, doi:10.1016/ S0165-4896 (03) 00077-5.

[2] Endre Boros, Vladimir Gurvich, Martin Milanič, Vladimir Oudalov \& Jernej Vičič (2018): A three-person deterministic graphical game without Nash equilibria. Discrete Applied Mathematics 243, pp. 21 - 38, doi:10.1016/j.dam.2018.01.008.

[3] Patricia Bouyer, Nicolas Markey \& Daniel Stan (2014): Mixed Nash Equilibria in Concurrent TerminalReward Games. In Venkatesh Raman \& S. P. Suresh, editors: FSTTCS 2014, Leibniz International Proceedings in Informatics (LIPIcs) 29, Schloss Dagstuhl-Leibniz-Zentrum fuer Informatik, pp. 351-363, doi:10 . 4230/LIPICs .FSTTCS.2014.351.

[4] Krishnendu Chatterjee \& Thomas A. Henzinger (2012): A survey of stochastic $\omega$-regular games. J. Comput. Syst. Sci 78(2), pp. 394-413, doi:10.1016/j.jcss.2011.05.002.

[5] Krishnendu Chatterjee, Rupak Majumdar \& Marcin Jurdzinski (2004): On Nash Equilibria in Stochastic Games. In Jerzy Marcinkowski \& Andrzej Tarlecki, editors: CSL 2004, Lecture Notes in Computer Science 3210, Springer, pp. 26-40, doi:10.1007/978-3-540-30124-0_6.

[6] H. Everett (1957): Recursive Games. In: Contributions to the Theory of Games Vol. III, Ann. Math. Studies 39, Princeton University Press, pp. 67-78, doi:10.1515/9781400882151-004.

[7] A. M. Fink (1964): Equilibrium in a stochastic n-person game. J. Sci. Hiroshima Univ. Ser. A-I Math. 28(1), pp. 89-93, doi:10.32917/hmj/1206139508.

[8] János Flesch, Frank Thuijsman \& O. J. Vrieze (1996): Recursive Repeated Games with Absorbing States. Math. Oper. Res 21(4), pp. 1016-1022, doi:10.1287/moor.21.4.1016.

[9] D. Gillette (1957): Stochastic games with zero stop probabilities. In: Contributions to the Theory of Games III, Ann. Math. Studies 39, Princeton University Press, pp. 179-187, doi:10.1515/9781400882151-011.

[10] Jeroen Kuipers, János Flesch, Gijs Schoenmakers \& Koos Vrieze (2009): Pure subgame-perfect equilibria in free transition games. European Journal of Operational Research 199(2), pp. 442 - 447, doi:10.1016/j . ejor.2008.11.038.

[11] Ashok P. Maitra \& William D. Sudderth (1998): Finitely additive stochastic games with Borel measurable payoffs. Int. J. Game Theory 27(2), pp. 257-267, doi:10.1007/s001820050071.

[12] Donald A. Martin (1975): Borel Determinacy. Annals of Mathematics 102(2), pp. 363-371, doi:10.2307/ 1971035.

[13] Donald A. Martin (1998): The Determinacy of Blackwell Games. J. Symb. Log 63(4), pp. 1565-1581, doi:10.2307/2586667.

[14] J.F. Mertens (1987): Repeated Games. In: Proceedings of the International Congress of Mathematicians, 1986, American Mathical Society, San Diego, pp. 1528-1577.

[15] J.F. Mertens \& A. Neyman (1981): Stochastic Games. Int. J. of Game Theory, pp. 53-66, doi:10.1007/ BF01769259. 
[16] Piercesare Secchi \& William D. Sudderth (2002): Stay-in-a-set games. Int. J. Game Theory 30(4), pp. 479490, doi:10.1007/s001820200092.

[17] L.S. Shapley (1953): Stochastic Games. Proc. Natl. Acad. Sci. U. S. A. 39, pp. 1095-1100, doi:10.1073/ pnas.39.10.1095.

[18] Robert Samuel Simon (2006): Value and perfection in stochastic games. Israel Journal of Mathematics 156(1), pp. 285-309, doi:10.1007/bf02773836.

[19] Masayuki Takahashi (1964): Equilibrium points of stochastic non-cooperative n-person games. J. Sci. Hiroshima Univ. Ser. A-I Math. 28(1), pp. 95-99, doi:10.32917/hmj/1206139509.

[20] Michael Ummels \& Dominik Wojtczak (2011): The Complexity of Nash Equilibria in Limit-Average Games. In Joost-Pieter Katoen \& Barbara König, editors: CONCUR 2011, Springer Berlin Heidelberg, pp. 482-496, doi:10.1007/978-3-642-23217-6_32.

[21] Nicolas Vieille (2000): Two-player stochastic games I: A reduction. Israel Journal of Mathematics 119(1), pp. 55-91, doi:10.1007/bf02810663.

[22] Nicolas Vieille (2000): Two-player stochastic games II: The case of recursive games. Israel Journal of Mathematics 119(1), pp. 93-126, doi:10.1007/bf02810664. 